\title{
Impact of multiple accelerometer IMU employment on the orientation estimate quality
}

\author{
Zdeněk Bouček ${ }^{1}$
}

\section{Introduction}

Orientation of quadrotor unmanned aerial vehicle (UAV) is essential for its navigation and stabilization. Vector of movement of helicopter in space is mainly based on sum of gravitational vector and vector of total thrust, which is generated by helicopter's rotors. Direction of vector of total thrust depends on UAV's orientation. Therefore the orientation has to be known with high precision.

Standard sensors, which are used for estimation of orientation in UAV applications, are microelectromechanical systems (MEMS) based triaxial digital accelerometers and gyroscopes, which are frequently used due to their low cost and small size. The accelerometer measures sum of static and dynamic acceleration. Under constant motion only the influence of Earth's gravitational field is reflected. The gyroscope measures the angular rate.

The goal is to analyze the influence of an extra accelerometer on the orientation estimate quality. The extra accelerometer measurement is more precise, however, it is designed with lower range.

\section{Orientation estimation}

Due to the nonlinear nature of the UAV's dynamic model and measurement function, the nonlinear estimator was employed. As the estimator the unscented Kalman filter was chosen. The nonlinearities of the system are handled with employment of unscented transform (in contrast to the extended Kalman filter, there is no need to calculate Jacobian matrices). In the prediction step in the UKF the UAV's dymamic model was used to achieve higher estimate precision. Estimated state consisted of orientation, angular rate and gyroscope bias.

\section{Evaluation of the additional accelerometer employment}

In the simulation the UAV carried out very aggressive maneuvers, where the helicopter was controlled to do one quick step in roll angle and than change it again to steady state, which caused movement of helicopter in sideways. In the same time the helicopter was commanded to do fast changes of pitch, which caused zigzag movement forwards and backwards. These fast changes in pitch angle continued for sixty seconds. During the whole flight the heading (yaw angle) was controlled with constant setpoint.

\footnotetext{
${ }^{1}$ student of doctoral study Applied Sciences and Informatics, field Cybernetics, e-mail: zboucek@students.zcu.cz
} 


\begin{tabular}{c|ccc}
\hline \multicolumn{4}{c}{ Aggressive maneuvers } \\
\hline Scenario & RMSE of roll & RMSE of pitch & RMSE of yaw \\
\hline deactivated & $5.9868 \cdot 10^{-5}$ & $6.4923 \cdot 10^{-5}$ & $1.1594 \cdot 10^{-3}$ \\
activated & $-14.2 \%$ & $-21.3 \%$ & $+0.001 \%$ \\
always on & $-14.2 \%$ & $-21.4 \%$ & $-0.04 \%$ \\
\hline \multicolumn{4}{c}{ Steady flight } \\
\hline deactivated & $5.9414 \cdot 10^{-5}$ & $5.9738 \cdot 10^{-5}$ & $1.1589 \cdot 10^{-3}$ \\
activated & $-14.2 \%$ & $-14.7 \%$ & $+0.005 \%$ \\
always on & $-14.2 \%$ & $-14.7 \%$ & $-0.06 \%$ \\
\hline
\end{tabular}

Table 1: RMSE for Euler angles

Simulation data were employed in testing of accelerometer settings in three scenarios:

1. Measurement from the extra accelerometer was not used.

2. Measurement from the extra accelerometer was used in its working range.

3. Measurement from the extra accelerometer was used even if the working range was exceeded. If the acceleration range is exceeded, output of accelerometer is cut to its minimal or maximal value in range.

\section{Experimental results}

Data for each scenario were evaluated in series of Monte Carlo simulations with the Root Mean Square error, see table 1. It can be seen that the best results were achieved in the scenario, where the accelerometer measurement was used even if the acceleration was out of range. The RMSE is lower due to the fact that the maximal acceleration during the flight was over the measurement range of the extra accelerometer, but still near to the real values.

The results imply that the extra accelerometer has a positive impact on the orientation estimate quality. The greatest impact is observable when the UAV performs aggressive maneuvers. Between the scenarios with the extra accelerometer there were not observed big differences. The second scenario is advantageous in the terms of saving the computational power and in application in the terms of saving the power-supply energy.

\section{Acknowledgement}

This publication was supported by the project LO1506 of the Czech Ministry of Education, Youth and Sports.

\section{References}

Duník, J., Šimandl, M., and Straka, O., 2012. Unscented Kalman Filter: Aspects and Adaptive Setting of Scaling Parameter. IEEE Transactions on Automatic Control, Vol. 57, pp 24112416.

Särkkä, S., 2013. Bayesian filtering and smoothing. Cambridge University Press. Institute of Mathematical Statistics textbooks. New York, USA.

Straka, O., Duník, J., and Šimandl, M., 2014. Unscented Kalman filter with advanced adaptation of scaling parameter. Automatica, Vol. 50. pp 2657-2664. 